Copyright by the Acoustical Society of America. Chatzipetros, A. A., Shaarawi, A. M., Besieris, I. M., \& Abdel-Rahman, M. A. (1998). Aperture synthesis of timelimited X waves and analysis of their propagation characteristics. Journal of the Acoustical Society of America, 103(5), 2287-2295. doi: 10.1121/1.422747

\title{
Aperture synthesis of time-limited $X$ waves and analysis of their propagation characteristics
}

\author{
Argyrios A. Chatzipetros \\ Paging Group, Motorola, Boyton Beach, Florida 33426
}

Amr M. Shaarawi

Department of Engineering Physics and Mathematics, Faculty of Engineering Cairo University, Giza, Egypt

loannis M. Besieris and Mohamed A. Abdel-Rahman

The Bradley Department of Electrical Engineering, Virginia Polytechnic Institute and State University, Blacksburg, Virginia 24061

(Received 20 June 1997; revised 8 January 1998; accepted 16 January 1998)

\begin{abstract}
The feasibility of exciting a localized X-wave pulse from a finite aperture is addressed. Also, the possibility of using a finite-time excitation of a dynamic aperture to generate a finite-energy approximation to an X-wave pulse is explored. The analysis is carried out by using a Gaussian time window to time limit the infinite $\mathrm{X}$-wave initial excitation. Huygens' construction is used to calculate the amplitude of the radiated wave field away from the finite-time source. The decay rate of the peak of the $\mathrm{X}$ wave is compared to that of a quasi-monochromatic signal. It is shown that the finite-time $\mathrm{X}$-wave propagates to much farther distances without significant decay. Furthermore, the decay pattern of the radiated $\mathrm{X}$-wave pulse is derived for a source consisting of an array of concentric annular sections. The decay behavior of the radiated pulse is similar to that of an X-wave launched from a finite-time aperture. This confirms the fact that time windowing the infinite energy $\mathrm{X}$-wave excitation is a viable scheme for constructing finite apertures. A discussion of the diffraction limit of the X-wave pulse is also provided. (c) 1998 Acoustical Society of America. [S0001-4966(98)00605-5]
\end{abstract}

PACS numbers: 43.20.Bi, 43.20.Px [JEG]

\section{INTRODUCTION}

In this paper, we address the possibility of exciting localized X-wave pulses from finite apertures. A large class of localized wave (LW) solutions to the scalar wave and Maxwell equations have been studied extensively. ${ }^{1-12}$ A number of these pulsed wave solutions are finite-energy variants of the focus wave mode (FWM). ${ }^{13,14}$ They are usually derived either as superpositions of infinite-energy FWM pulses, ${ }^{8-11,14}$ or by time limiting various FWM-like excitations to construct finite-energy aperture fields. ${ }^{2-7}$ Alternatively, such LW pulses can be synthesized as superpositions over Bessel beams with appropriately chosen spectra. ${ }^{15}$ Our approach is based mainly on the proven possibility of exciting Bessel beams from infinite apertures. ${ }^{1-4}$ It has been shown that a Bessel beam defined initially on an infinite plane (e.g., $z=0$ ) propagates under the effect of Huygens' operator in the positive $z$ direction. ${ }^{1-4}$ Any possible contributions from acausal (negative $z$-directed) components sum up to zero.

In contradistinction to other solutions excited from infinite apertures, such as plane waves and Bessel beams, the excitation of the FWM pulse does not require infinite power. This is the case because as the generating aperture becomes infinitely large, the intensity of the field exciting it decreases to zero as $(c t)^{-2}$, while the area of the aperture increases as $(c t)^{2}$. These two effects balance each other and the power of the excitation wave field remains constant. The energy of the generated pulse becomes infinite because the aperture is illuminated for an infinitely long time. Time limiting the ex- citation wave field results in the generation of finite-energy LW pulses.

Other pulsed solutions exhibiting distinct similarities to FWM-like pulses are the $\mathrm{X}$-wave pulses introduced by $\mathrm{Lu}$ and Greenleaf. ${ }^{16-23}$ Similarly to the FWM-like pulses, the $\mathrm{X}$-waves have extended ranges of localization and acquire large focus depths in the near field. These properties make these pulsed wave fields ideal for applications involving detection of objects buried at unknown depths, as well as in high-resolution imaging. The use of $\mathrm{X}$-wave pulses in ultrasonic medical imaging has been investigated by $\mathrm{Lu}$ and Greenleaf. ${ }^{16-23}$ They have shown that X-waves can be generated using ultrasonic transducers consisting of annular arrays of concentric piezoelectric rings excited by independent waveforms applied at different times (Refs. 16, 18; cf. also Ref. 24). Furthermore, Lu and Greenleaf have studied experimentally the improvement in pulse-echo imaging using $\mathrm{X}$-waves. ${ }^{21}$ Applying $\mathrm{X}$-waves to tissue-equivalent phantoms and standard test objects, they have shown that resolution of pulse-echo images obtained using $\mathrm{X}$-waves are superior to that obtained with focused Gaussian beams. ${ }^{21,22}$ In addition to their large focus depths, X-waves and FWM-like pulses are both wideband wave fields. This property renders them suitable for applications involving parameter characterization using backscattered signals from objects of varying sizes. Along this direction, Donnelly and Power ${ }^{25}$ have shown that an acoustical LW pulse backscattered from compressible spheres immersed in water can accurately provide information about the materials as well as the sizes of the spheres. 
Similar results can be obtained using $\mathrm{X}$-wave acoustical pulses. As a consequence, these pulsed wave fields can have applications in parameter characterization and nondestructive testing of materials.

The excitation wave field of an $\mathrm{X}$-wave pulse has the form of an annulus whose radius varies with time. The speed of expansion $v_{\text {ap }}$ of the aperture field is related to the speed of the pulse center $v_{p}$ and can be greater than the wave speed $c$ of the medium. The temporal and spatial frequency content of an X-wave pulse will be calculated in order to improve our understanding of the decay pattern of the radiated wavefield. Similarly to the FWM-like solutions, we shall explore the possibility of using a finite-time excitation of an X-wave by imposing a Gaussian time window on the infinite time initial X-wave field. The amplitude of the radiated pulse will be calculated in the near-to-far field range and its decay pattern will be compared to that of a quasi-monochromatic pulse characterized by a specific carrier frequency. It will be shown that the finite-time version of the $\mathrm{X}$-wave propagates without significant decay much further than quasimonochromatic signals.

\section{THE X-WAVE APERTURE}

The $\mathrm{X}$-wave solution to the 3-D scalar wave equation may be expressed as

$$
\Psi_{X W}(\rho, z, t)=\frac{z_{0}}{\left[\left(z_{0} \rho\right)^{2}+\left(a_{1} z_{0}+i(z-c t \operatorname{coth} \beta)\right)^{2}\right]^{1 / 2}},
$$

where $a_{1}$ and $\beta$ are free real parameters and $z_{0}=1 / \sinh \beta$. Note that $c \operatorname{coth} \beta$ is the propagation speed of the pulse. To ensure that the field will propagate in the forward direction, we restrict $\beta$ to be positive. The solution given in Eq. (1) is identical to that derived by $\mathrm{Lu}$ and Greenleaf ${ }^{16,17}$ if the following substitutions are made: $a_{1} z_{0} \rightarrow a_{0} / \cos \eta, \quad z_{0} \rho$ $\rightarrow \rho \tan \eta$ and $c$ coth $\beta \rightarrow c / \cos \eta$. In the notation used by Lu and Greenleaf, ${ }^{16} \alpha_{0}$ is a real parameter and $\eta$ is the axicon angle.

The infinite energy solution given in Eq. (1) was used in Ref. 16 to excite an aperture of a finite size situated at $z$ $=0$ and then to compute the radiated field. In this paper, we construct a finite aperture in a different manner. We use the fact that the $\mathrm{X}$-wave excitation at $z=0$ acquires the form of an annulus whose radial position is time dependent. By time limiting the excitation wave field, we restrict the radius of the time-dependent annulus to be smaller than a finite maximum value. The advantage of such an approach is that the spatio-temporal spectral structure of the radiated pulse becomes very transparent. One can then have a better understanding of the depletion of the spectral content of such a pulse, as well as its behavior as it decays away from its source. ${ }^{2,4-7}$ Subsequently, this information can be used to design other localized variants of $X$ waves. First, we shall present some of the aspects of the $\mathrm{X}$-wave excitation given in Eq. (1) without time-limiting it. In Sec. II, the finite-time $\mathrm{X}$-wave will be studied in detail.

Consider the $\mathrm{X}$-wave excitation of a planar aperture positioned at $z=0$, viz.,

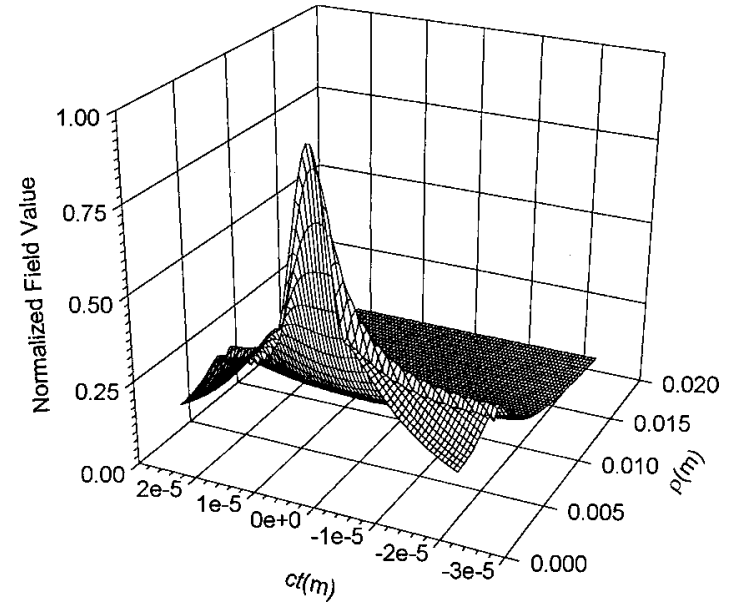

FIG. 1. Surface plot of the real part of the $\mathrm{X}$-wave pulse on the aperture plane $(z=0)$ for the parameters $a_{1}=0.004 \mathrm{~m}^{-1}, \quad \beta=7.4$, and $c$ $=1500 \mathrm{~m} / \mathrm{s}$.

$$
\Psi_{X W}(\rho, z=0, t)=\frac{1}{\left[\rho^{2}+\left(a_{1}-i y_{0} t\right)^{2}\right]^{1 / 2}},
$$

where $y_{0}=c \cosh \beta$. The surface plot of the real part of the aperture field given in (2) is shown in Fig. 1 for $a_{1}$ $=0.004 \mathrm{~m}$ and $\beta=7.4$. The wave velocity of the medium is chosen to be $c=1500 \mathrm{~m} / \mathrm{s}$. The intensity of the infinite energy excitation of an $\mathrm{X}$-wave aperture is given as

$$
\begin{aligned}
I(\rho, 0, t) & =\Psi(\rho, 0, t) \Psi^{*}(\rho, 0, t) \\
& =\frac{1}{\left[\left(\rho^{2}-\left(y_{0} t\right)^{2}\right)^{2}+a_{1}^{4}+2 a_{1}^{2} \rho^{2}+2 a_{1}^{2}\left(y_{0} t\right)^{2}\right]^{1 / 2}} .
\end{aligned}
$$

After rearrangement, the denominator in (3) becomes

$$
\begin{aligned}
D= & {\left[\rho^{4}+\left(y_{0} t\right)^{4}-2 \rho^{2}\left(y_{0} t\right)^{2}+a_{1}^{4}+2 a_{1}^{2} \rho^{2}\right.} \\
& \left.+2 a_{1}^{2}\left(y_{0} t\right)^{2}\right]^{1 / 2},
\end{aligned}
$$

and for $\rho \gg a_{1}$

$$
D \cong\left[\rho^{4}+\left(y_{0} t\right)^{4}-2 \rho^{2}\left(y_{0} t\right)^{2}\right]^{1 / 2}=\left[\rho^{2}-\left(y_{0} t\right)^{2}\right] .
$$

Expressions (3) and (5) show how the intensity of the illumination wave field varies with time at the various radial positions $\rho$ on the aperture plane. At the centroid of the excitation field $(\rho=0$ and $t=0)$ the intensity $I$ is finite and is equal to $1 / a_{1}^{2}$. For large values of $\rho$ and $t$, the highest intensity occurs at $\rho=y_{0} t$. Thus, the illumination of the X-wave aperture acquires the form of an annulus whose radius changes linearly with time. The intensity at $\rho=y_{0} t$ varies as $1 / a_{1} \sqrt{a_{1}^{2}+4 \rho^{2}}$, as it can be deduced from Eq. (3). Since the area of an annulus is proportional to $\rho$, it follows that the power of the initial X-wave on the aperture (=intensity $\times$ area) remains constant as $c t \rightarrow \infty$. The energy of the excitation wave field is infinite because the aperture is illuminated for an infinite time duration.

The time dependence of the radius of the X-wave aperture is found from the real part of Eq. (2). The amplitude of the real part is maximum on the aperture at $\rho=0$ and $t=0$. The highly focused central portion of the aperture has a ra- 


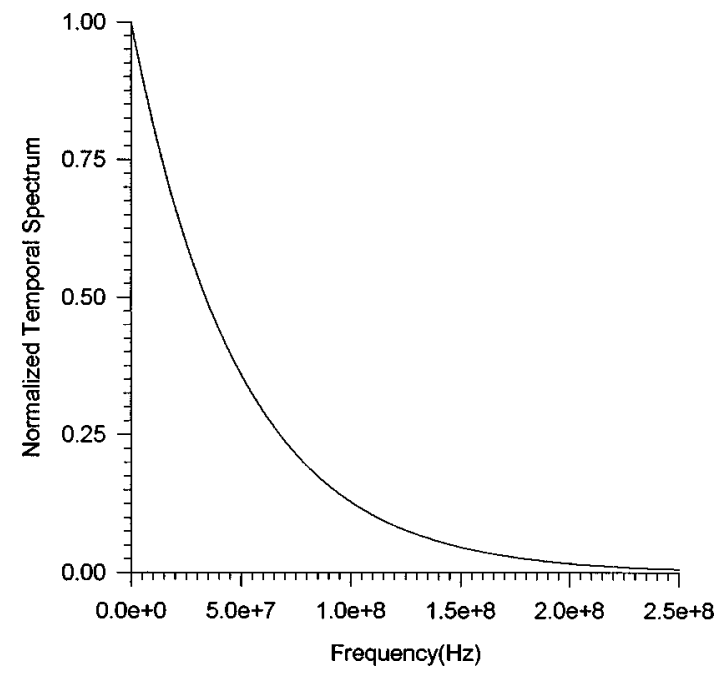

FIG. 2. Temporal frequency content, $\Phi_{t}(\rho, \omega)$, of the X-wave pulse at $\rho$ $=0$ for the parameters $a_{1}=0.004 \mathrm{~m}^{-1}, \beta=7.4$, and $c=1500 \mathrm{~m} / \mathrm{s}$.

dius $R_{\min }=R(t=0)=a_{1}$. As $y_{0} t$ becomes larger than $a_{1}$, the annulus forms at $R(t)=y_{0} t$ with a width of order $a_{1}$ $\ll R(t)$. As a consequence, a good estimate of the radius of the excitation wave field as a function of time is given by

$$
R(t)=y_{0} t+R(0) .
$$

For $y_{0} t \gg a_{1}$, the speed of the expansion of the aperture is given by $v_{\text {ap }}=y_{0}=c \cosh \beta$. The speed of the centroid of the radiated pulse and that of the expansion of the aperture are always greater than $c$. However, the faster the centroid of the pulse travels, the slower the expansion of the aperture becomes.

\section{FREQUENCY SPECTRA OF THE X-WAVE}

In order to gain a better understanding of the spectral content of the X-wave pulse, we need to define the temporal and spatial frequency contents of the excitation wave field given in Eq. (2). Both spectra have to be studied because the $\mathrm{X}$-wave illumination is a pulsed wave field exciting different parts of the generating aperture at different times. The spectrum of the initial excitation given in Eq. (2) is deduced from the Fourier inversion

$$
\begin{aligned}
\Phi(\chi, \omega)= & \int_{-\infty}^{+\infty} d t \int_{0}^{+\infty} d \rho \rho J_{0}(\chi \rho) \\
& \times e^{-i \omega t} \frac{1}{\left[\rho^{2}+\left(a_{1}-i y_{0} t\right)^{2}\right]^{1 / 2}} .
\end{aligned}
$$

The integration over $\rho$ is carried out using (6.554.1) of Ref. 26 ; as a consequence, we obtain

$$
\Phi(\chi, \omega)=\frac{1}{\chi} \int_{-\infty}^{+\infty} d t e^{-i \omega t} e^{-\chi\left(a_{1}-i y_{0} t\right)} .
$$

The integration over $t$ yields the following Fourier spectrum:

$$
\Phi(\chi, \omega)=\frac{2 \pi}{\chi} e^{-\chi a_{1}} \delta\left(\omega-\chi y_{0}\right) .
$$

One should note that the argument of the Dirac $\delta$ function couples the temporal frequency $\omega$ to the spatial wave number

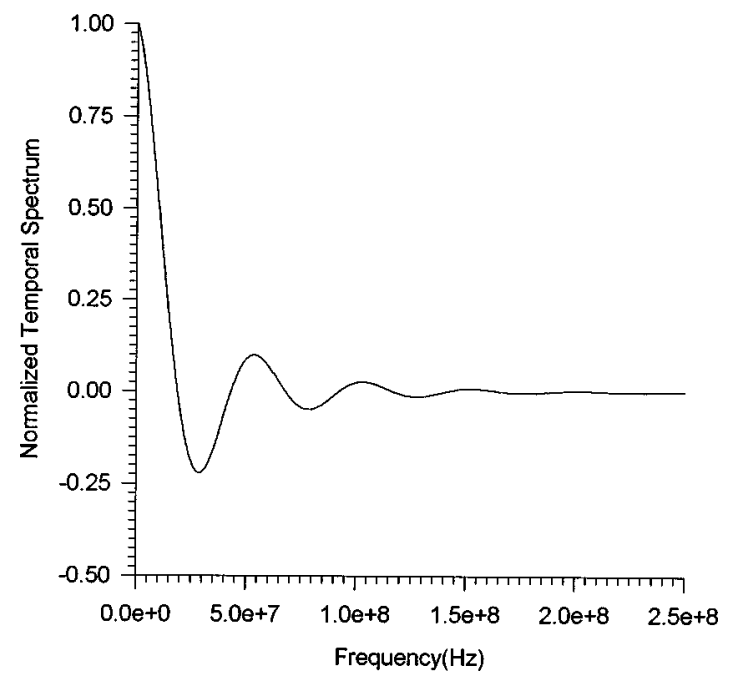

FIG. 3. Temporal frequency content, $\Phi_{t}(\rho, \omega)$, of the X-wave pulse at $\rho$ $=2.5 \mathrm{~cm}$ for the parameters $a_{1}=0.004 \mathrm{~m}^{-1}, \beta=7.4$, and $c=1500 \mathrm{~m} / \mathrm{s}$.

$\chi$. This is a characteristic attribute of most LW pulses. A similar $\delta$ function appears in the Fourier spectrum of the infinite-time FWM excitation of a flat aperture studied in Refs. 1 and 2. For the FWM spectrum we have $\omega \propto \chi^{2}$, while the X-wave exhibits an $\omega \propto \chi$ coupling. The appearance of a Dirac $\delta$ function in the spectrum signifies that the initial wave field illuminates the aperture for an infinite time.

To identify the temporal frequency content of the excitation wave field at various positions on the aperture we define the temporal spectrum as follows:

$$
\begin{aligned}
\Phi_{t}(\rho, \omega) & =\int_{0}^{\infty} d \chi \chi J_{0}(\chi \rho) \Phi(\chi, \omega) \\
& =2 \pi \int_{0}^{\infty} d \chi \chi J_{0}(\chi \rho) \frac{e^{-\chi a_{1}}}{\chi} \delta\left(\omega-\chi y_{0}\right)
\end{aligned}
$$

The above integration gives

$$
\Phi_{t}(\rho, \omega)=\frac{2 \pi}{y_{0}} J_{0}\left(\omega \rho / y_{0}\right) e^{-\omega a_{1} / y_{0}} \mathbf{H}_{\mathbf{s}}(\omega),
$$

where $\mathbf{H}_{\mathbf{s}}(\omega)$ is the Heaviside unit-step function. The highest contribution to the temporal frequency content comes from values close to $\omega=0 \mathrm{rad} / \mathrm{s}$. The maximum frequency is determined from the exponential and is given explicitly as

$$
\omega_{\max }=\left(4 y_{0} / a_{1}\right)=\left(4 c \cosh \beta / a_{1}\right) .
$$

For the parameters $a_{1}=0.004 \mathrm{~m}, \beta=7.4$, and $c=1500 \mathrm{~m} / \mathrm{s}$, the maximum frequency becomes $\omega_{\max }=1.227 \times 10^{9} \mathrm{rad} / \mathrm{s}$ or $f_{\max }=195.3 \mathrm{MHz}$. This implies that the minimum wavelength in this case is $\lambda_{\min }=1.54 \mathrm{~m}$. The temporal spectrum given in Eq. (11) is plotted in Fig. 2 for $\rho=0$. The same spectrum is shown in Fig. 3 at $\rho=0.025 \mathrm{~m}$. The oscillations appearing in the latter case indicate that an integration over $\omega$ should yield a smaller amplitude. This is consistent with the field given in Eq. (2), where the amplitude is maximum at $\rho=0$ and decreases as $\rho$ becomes larger. 


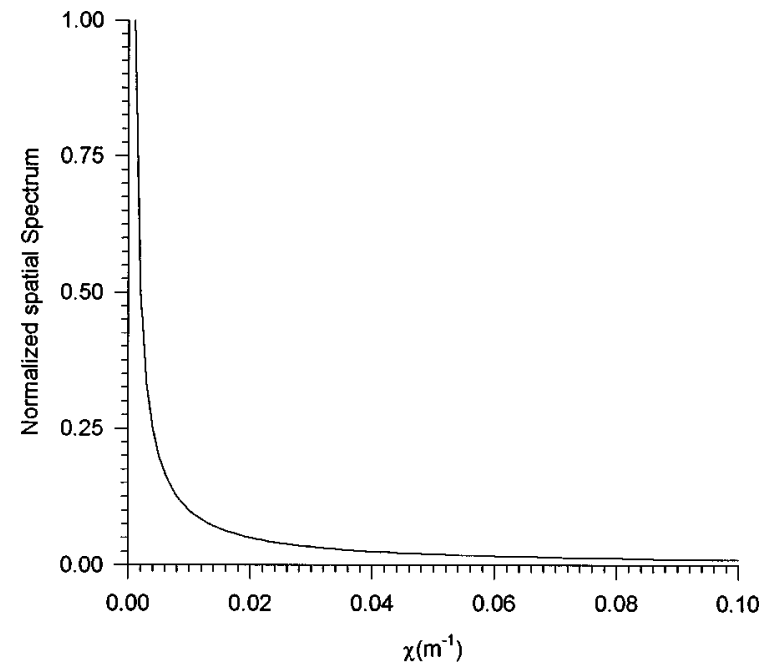

FIG. 4. Spatial frequency content, $\Phi_{s}(\chi, t)$, of the $\mathrm{X}$-wave pulse at $t=0$ for the parameters $a_{1}=0.004 \mathrm{~m}^{-1}, \beta=7.4$, and $c=1500 \mathrm{~m} / \mathrm{s}$.

The spatial spectrum, on the other hand, describes the spatial distribution of the source elements on the aperture plane for various times. We define the spatial spectrum as

$$
\Phi_{s}(\chi, t)=\frac{1}{2 \pi} \int_{0}^{\infty} d \omega e^{i \omega t} \frac{2 \pi}{\chi} e^{-\chi a_{1}} \delta\left(\omega-\chi y_{0}\right)
$$

or

$$
\Phi_{s}(\chi, t)=\frac{1}{\chi} e^{-\chi a_{1}} e^{i \chi y_{0} t}
$$

The spectrum given in Eq. (14) is shown in Figs. 4 and 5 for $t=0$ and $t=5 \times 10^{-4} \mathrm{~s}$, respectively. The oscillations appearing in the latter case indicate that the amplitude of the $X$ wave decreases after the highly focused central portion of the pulse has been launched from the aperture.

\section{FINITE TIME EXCITATION}

We have shown in Sec. I that the power required to excite an $\mathrm{X}$-wave aperture is always finite and that the excitation energy is infinite simply because the source is excited for an infinite duration of time. As a consequence, the excitation wave field, having the shape of an annulus, is allowed to expand to infinite dimensions. The illumination given in Eq. (2) thus requires an infinite aperture to be realized. Because of the time dependence of the radius of the significant annular contributions to the excitation wave field, we can construct a finite-energy source using one of the following two methods. First we can apply the $\mathrm{X}$-wave excitation given in Eq. (2) to an aperture having a finite radius $R$ and calculate the amplitude of the pulse radiated into the $z>0$ halfspace. $\mathrm{Lu}$ and Greenleaf ${ }^{16}$ used this method to construct a finite $X$-wave aperture. One should note that at some instant in time we will have $R(t)>R$; subsequently, the excitation wave field inside the finite radius of the aperture becomes very small and can be neglected for all practical purposes. One can then presume that the illumination wave field is turned off at that time instant. Alternatively, we can start by time limiting the infinite excitation given in Eq. (2). This allows the radius of the annular excitation to expand to a

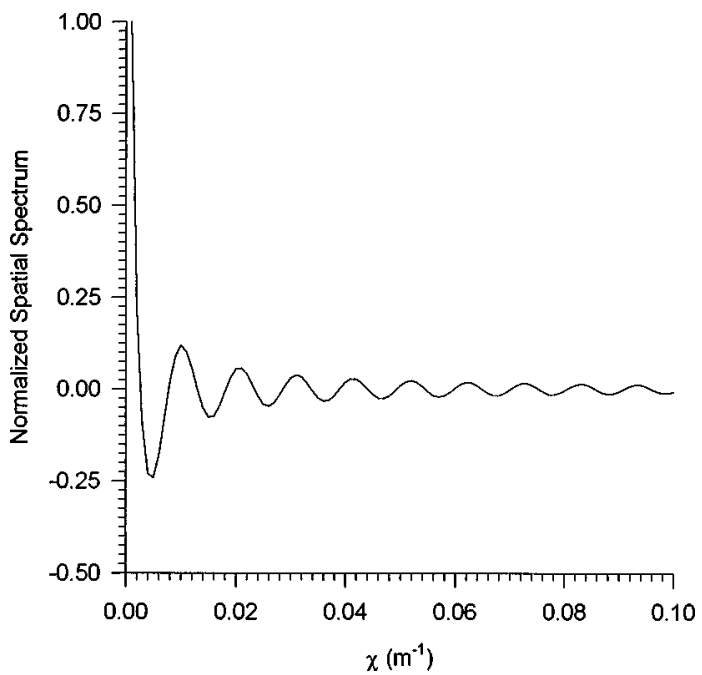

FIG. 5. Spatial frequency content, $\Phi_{s}(\chi, t)$, of the X-wave pulse at $t=5$ $\times 10^{-4} \mathrm{~s}$ for the parameters $a_{1}=0.004 \mathrm{~m}^{-1}, \beta=7.4$, and $c=1500 \mathrm{~m} / \mathrm{s}$.

finite radius before the initial wave field is turned off. The same approach has been used extensively in defining finite FWM excitations. ${ }^{2,3,5,6}$ The latter approach has the advantage of providing a deeper insight into the spectral structure of the radiated LW pulses. Within such a framework, we have been able to demonstrate that the spectral depletion of LW pulses is essentially different from that of the usual quasimonochromatic pulses. ${ }^{6}$ This spectral approach has also helped us in deriving a diffraction-limit formula capable of characterizing the decay behavior of ultra-wideband LW pulses. $^{7}$ Because of these advantages, we seek to use this approach to time limit the X-wave excitation (2), thus constructing a finite-energy $\mathrm{X}$-wave aperture. The decay patterns of the radiated fields calculated using both approaches will be compared and the differences between the two methods will be discussed.

A convenient time window to use is the Gaussian exp $\left(-t^{2} / 4 T^{2}\right)$. In this case, the Fourier spectrum of the initial excitation can be calculated as follows:

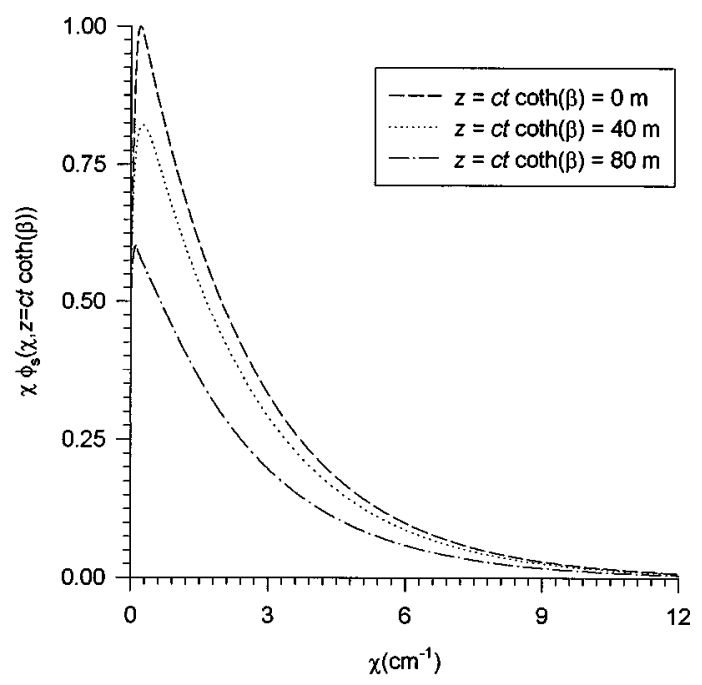

FIG. 6. Normalized spatial spectrum of the finite-time $X$ wave at different distances $z=c t$ coth $\beta=0,40$, and $80 \mathrm{~m}$ for $R_{\max }=0.15 \mathrm{~m}$. 


$$
\begin{aligned}
\Phi(\chi, \omega)= & \int_{-\infty}^{+\infty} d t \int_{0}^{+\infty} d \rho \rho J_{0}(\chi \rho) \\
& \times e^{-i \omega t} \frac{e^{-t^{2} / 4 T^{2}}}{\left[\rho^{2}+\left(a_{1}-i y_{0} t\right)^{2}\right]^{1 / 2}} .
\end{aligned}
$$

The integration over $\rho$ is carried out using (6.554.1) of Ref. 26 , yielding

$$
\Phi(\chi, \omega)=\frac{e^{-\chi a_{1}}}{\chi} \int_{-\infty}^{+\infty} d t e^{-i t\left(\omega-\chi y_{0}\right)} e^{-t^{2} / 4 T^{2}}
$$

or

$$
\Phi(\chi, \omega)=\frac{2 \pi}{\chi} e^{-\chi a_{1}} \hat{\delta}\left(\omega-\chi y_{0}\right)
$$

where $\hat{\delta}\left(\omega-\chi y_{0}\right)$ is a Gaussian function given explicitly by

$$
\hat{\delta}\left(\omega-\chi y_{0}\right)=\frac{T}{\sqrt{\pi}} e^{-T^{2}\left(\omega-\chi y_{0}\right)^{2}} .
$$

Note that the parameter $T$ characterizes the cutoff time of the illumination of the aperture. As a consequence, it determines the maximum radius of the aperture, i.e., the maximum radius of the annular illumination before the field is cutoff. Even though for a Gaussian window we cannot define a precise cutoff time, it is convenient to use such a function because its Fourier transform is a smooth Gaussian. A rectangular time window provides a definite cutoff time, but its Fourier transform is highly oscillatory and more spread out than the Gaussian $\omega$-window given in Eq. (17b). As a consequence, the Gaussian time window is easier to handle numerically, while its $e^{-2}$ point provides a reasonable estimate of the cutoff time. The use of other time windows and their effect on the decay patterns of FWM-like pulses have been investigated. ${ }^{5,7}$ We have shown that the decay of the centroid of LW pulses can be altered significantly in the near-to-farfield range by changing the time sequence of the initial excitation. We believe that similar results can be obtained for finite-time $\mathrm{X}$-wave apertures. For large $T$ values, the Gaussian $\omega$ window in $(17 \mathrm{~b})$ reduces to a narrow distribution centered around $\omega \sim \chi y_{0}=\chi c \cosh \beta$. The spectral width of such a window is chosen to be smaller than $\omega_{\max }$. Therefore, the temporal spectrum can be viewed as a juxtaposition of these windows centered around all the significant $\chi$ components determined by the spatial spectrum given in Eq. (14).

Using Huygen's formula, ${ }^{27}$ the field radiated into the $z$ $>0$ half-space can be expressed as ${ }^{1-3}$

$$
\begin{aligned}
\Psi(\rho, z, t)= & \int_{0}^{+\infty} d \chi \chi J_{0}(\chi \rho) \\
& \times \int_{0}^{+\infty} d \omega \Phi(\chi, \omega) e^{i \omega t} e^{-i \sqrt{(\omega / c)^{2}-\chi^{2}} z}
\end{aligned}
$$

Substituting the spectrum given in Eq. (17a), the amplitude of the radiated field may be expressed as a superposition over the spatial spectral content of the X-wave pulse; specifically,

$$
u(\rho, z, t)=\mathfrak{R} e\{\Psi(\rho, z, t)\}
$$

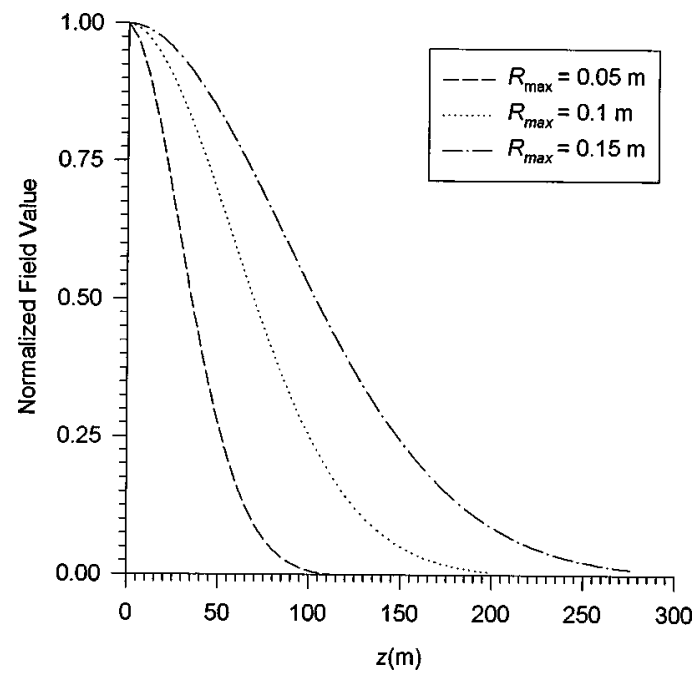

FIG. 7. Decay pattern of the centroid of the finite time $X$ wave for different values of the maximum aperture radius.

$$
=\int_{0}^{+\infty} d \chi \chi J_{0}(\chi \rho) \hat{\Phi}_{s}(\chi, z, t) .
$$

Here, $\hat{\Phi}_{s}(\chi, z, t)$ is the spatial spectral content at a distance $z$ and time $t$. It is given explicitly by

$$
\begin{aligned}
\hat{\Phi}_{s}(\chi, z, t)= & \int_{0}^{+\infty} d \omega \frac{2 \pi}{\chi} e^{-\chi a_{1}} \hat{\delta}\left(\omega-\chi y_{0}\right) \\
& \times \cos \left(\sqrt{(\omega / c)^{2}-\chi^{2}} z-\omega t\right) .
\end{aligned}
$$

For the field generated from an infinite aperture $(T \rightarrow \infty)$, the Gaussian in Eq. (19) becomes a Dirac $\delta$ function forcing $\omega$ to equal $\chi y_{0}$. In this limit, the argument of the sinusoidal term becomes equal to $\chi \sinh \beta(z-c t \operatorname{coth} \beta)$, leading to the diffraction-free $\mathrm{X}$ wave moving with speed $v_{p} \equiv c \operatorname{coth} \beta$. For the finite-time excitation, the fine spectral balance that yields the exact $(z-c t$ coth $\beta$ ) functional dependence is upset. Instead, as the pulse travels away from the source plane, the sinusoidal term progressively introduces oscillations into the Gaussian windows $\hat{\delta}\left(\omega-\chi y_{0}\right)$ centered around all the significant spectral $\chi$ components. ${ }^{3,5-7}$ Since these oscillations increase with distance, the integration over $\omega$ yields smaller spectral contributions at the different $\chi$ frequencies. As a consequence, the integration over $\chi$ in Eq. (19a) gives a field amplitude that decreases with distance. The depletion of the spatial spectral components of the $X$ wave is depicted in Fig. 6, where we have plotted the spatial spectrum given in Eq. (19b) for $c T=91.69 \mu \mathrm{m}$ and at $z=c t$ coth $\beta=0,40$, and $80 \mathrm{~m}$. This figure shows that the spatial spectral components acquire smaller amplitudes as the pulse travels away from the aperture. Consequently, the integration over $\chi$ in Eq. (19) yields the expected decay pattern of the centroid of the radiated pulse. The decay of the peak $(\rho=0$ and $z=c t \operatorname{coth} \beta)$ of the $\mathrm{X}$ wave, normalized with respect to the peak amplitude at the aperture, is shown in Fig. 7. The curves are plotted for the cutoff times $c T=30.56,61.13$, and $91.69 \mu \mathrm{m}$. Such cutoff times correspond to time-dependent apertures acquiring maximum radii $R_{\max }=R(2 c T)=5,10$, and $15 \mathrm{~cm}$. 


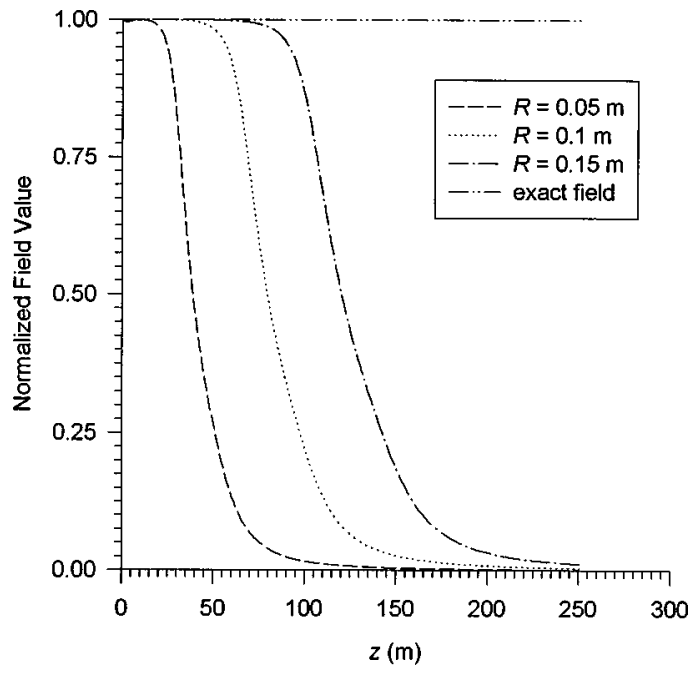

FIG. 8. Decay pattern of the centroid of the X-wave pulse generated by an array of concentric annular sections for different radii.

To verify the validity of the estimated maximum radii, we deduce the decay pattern of the peak of the $\mathrm{X}$-wave using a finite-radius aperture. For such a construction the source is assumed to consist of a large number of concentric annular array elements. The amplitude of the initial excitation is specified on each distinct element and the radiated field along the axis of propagation $(\rho=0)$ is evaluated using the following discrete version of Huygens' formula:

$$
\begin{aligned}
u_{H}(0, z, t)= & -\sum_{n=1}^{N} \frac{A_{n}}{4 \pi R_{n}}\left\{\left[\partial_{z^{\prime}} u\right]-\left[\partial_{c t^{\prime}} u\right] \frac{z-z_{0}}{R_{n}}\right. \\
& \left.-[u] \frac{z-z_{0}}{R_{n}^{2}}\right\} .
\end{aligned}
$$

Here, $A_{n}$ is the area of the annular section labeled $n, N$ is the total number of annular sections, $\rho_{n}^{\prime}=n \Delta \rho^{\prime}$ specifies the radial position of the annular section labeled $n$, and $z$ is the distance at which the field of the radiated pulse is reconstructed. The distance from the source point to the observation point is denoted by $R_{n}=\sqrt{\left(n \Delta \rho^{\prime}\right)^{2}+\left(z-z_{0}\right)^{2}}$ for $\rho=0$. The square brackets in Eq. (20) indicate that the enclosed quantities are evaluated at the retarded time $t^{\prime}=t-\left(R_{n} / c\right)$. The main purpose of the numerical reconstruction procedure embodied in Eq. (20) is to focus on the possibility of using space-time sources on the aperture plane $z=0$ in order to generate LW pulses in the region $z \geqslant 0$. A second objective is to gain more physical insight into the propagation characteristics of the reconstructed pulses.

For annular elements of width $\Delta \rho^{\prime}=1 \mathrm{~mm}$, we can construct apertures having radii $R=5,10$, and $15 \mathrm{~cm}$ by using $N=50,100$, and 150 sections, respectively. The decay patterns of the LW pulses radiated by these three apertures are plotted in Fig. 8 for the values of $a_{1}$ and $\beta$ used earlier. A comparison between Figs. 7 and 8 shows that the ranges of the two decay patterns are similar. For the finite-time dynamic apertures, the centroids of the radiated $\mathrm{X}$ waves start decaying at close ranges and their rolloff is smoother than that of $\mathrm{X}$ waves generated from discrete circular arrays. The centroids of the $\mathrm{X}$-wave pulses generated by discrete circular

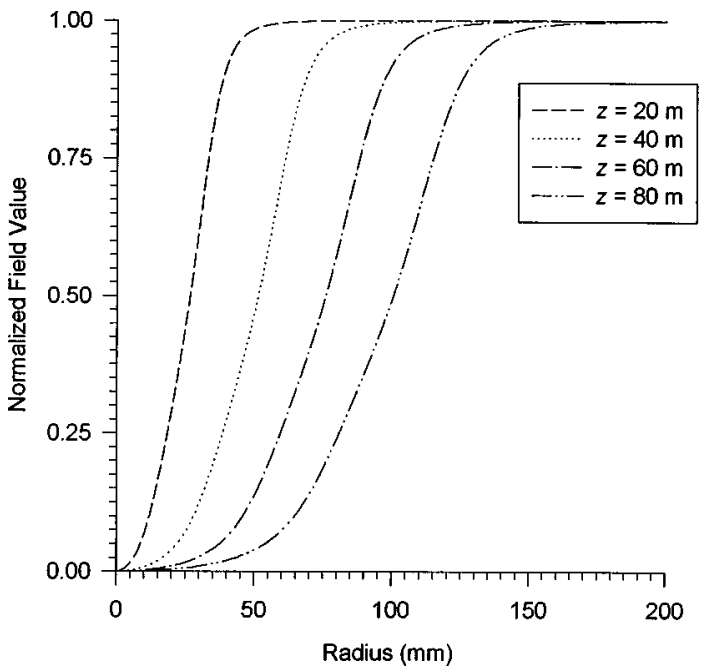

FIG. 9. Contribution of the different annular sections to the field amplitudes at various distances.

arrays exhibit a very slow decay in the near field. The decay in the far field, however, is more abrupt than that corresponding to the finite-time pulses. The smoother decay is attributed to the continuous turnoff of the initial finite-time excitation due to the application of the Gaussian time window. The excitation wave field, therefore, acquires smaller amplitudes at larger radial positions as the illumination of the aperture approaches its turn off point. We would like to emphasize, however, that the finite-time aperture performance is very close to that of a physically bounded source even though the former has no physical edge containing the illumination wave field. This is a crucial point allowing us to use finite-time dynamic sources in our analysis instead of physical apertures. The former lend themselves nicely to the advocated spectral depletion approach. In Fig. 9 we display a set of curves showing how the amplitude of the peak of the propagating $\mathrm{X}$ wave, at a specific distance, consists of contributions from the illumination of a finite domain of the aperture. This information can be used to determine the size of the aperture needed to radiate a decay-free pulse up to a specific distance $z$ (cf. Ref. 20 for an experimental procedure along this path).

Finally, we provide in Figs. 10 and 11 a comparison between the shapes of the longitudinal envelopes of the propagating $X$ waves at $z=100$. In Fig. 10 we display the time history of the $\mathrm{X}$-wave pulse launched from a finite-time aperture and compare it to the initial pulse excitation at the aperture. On the other hand, in Fig. 11 we compare an Xwave pulse generated by a discrete circular array to the exact wave solution. The oscillations appearing in the front part of the radiated $\mathrm{X}$ wave are due to the discretization of the excitation wave field. Otherwise, the time histories of the pulses generated using the two suggested frameworks are almost identical to the exact wave solution.

\section{DIFFRACTION LENGTH ANALYSIS}

For a quasi-monochromatic $\mathrm{CW}$ signal, the diffraction length is determined in terms of the Rayleigh distance $\pi R^{2} / \lambda_{c}$, where $R$ is the radius of the source and $\lambda_{c}$ is the 


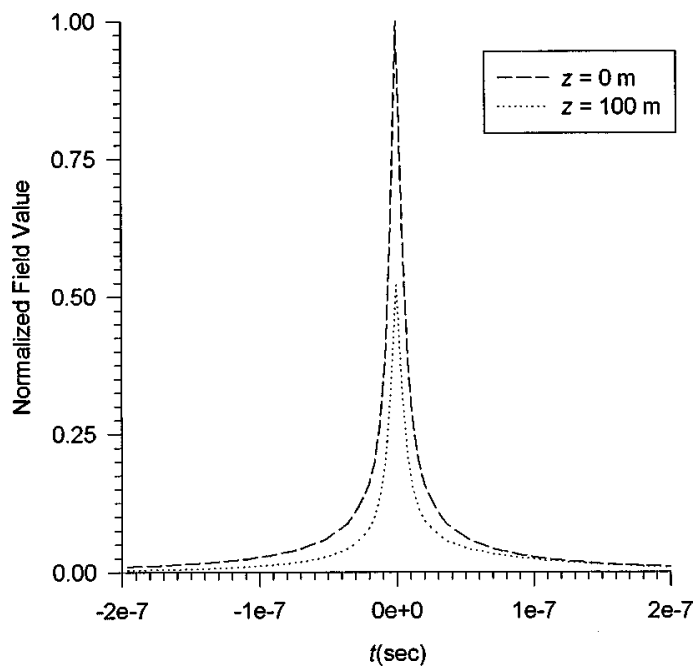

FIG. 10. The time history of the finite-time $X$-wave pulse at $z=0,100 \mathrm{~m}$ due to an aperture having radius $R_{\max }=0.15 \mathrm{~m}$.

wavelength of the carrier wave field. In contradistinction, any attempt to define the diffraction length of a LW pulse generated by a dynamic aperture antenna is more involved. The difficulty can be attributed to the ultra-wideband temporal bandwidth of the LW pulse, as well as the fact that the aperture is characterized by a time-varying radius. One of the earlier attempts to define a diffraction length for LW pulses generated by dynamic aperture antennas was undertaken by Hafizi and Sprangle. ${ }^{28}$ Their diffraction length is given as $Z_{\mathrm{HS}}=\left(2 \pi w_{0} R_{\max }\right) / \lambda_{\min }$, where $w_{0}$ is the beam waist, $R_{\max }$ is the maximum dimension of the aperture or antenna, and $\lambda_{\min }$ is the minimum wavelength. Specific applications of this formula to FWM-like LWs can be found in Ref. 7.

The Hafizi-Sprangle diffraction length gives a good broad sense estimate of the behavior of LW wave pulses as they propagate away from their generating aperture planes. However, it does not include special local features arising from different types of dynamic aperture excitations. In this section, the decay pattern of an X-wave pulse will be char-

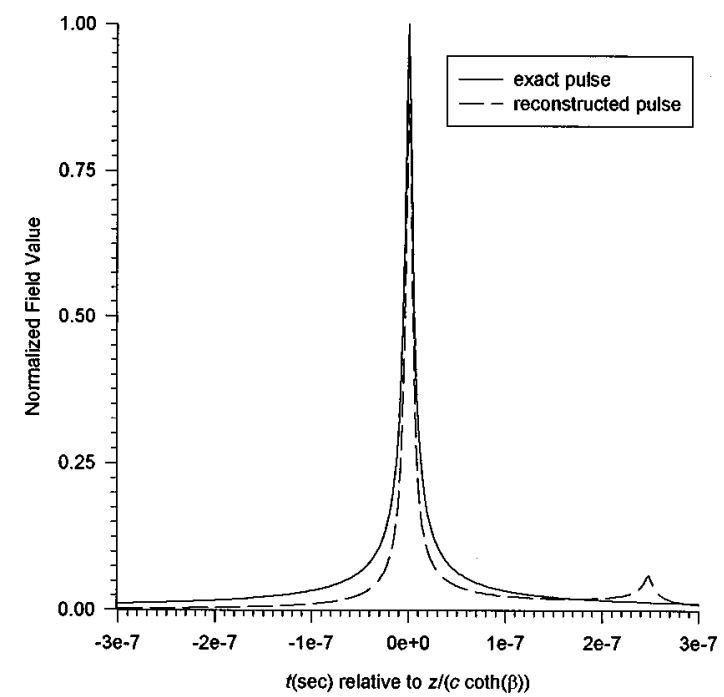

FIG. 11. The time history of the X-wave pulse reconstructed at $z=100 \mathrm{~m}$ from a circular array and compared to the exact solution at the same distance. acterized using a diffraction length criterion that has been proposed recently. ${ }^{7}$ Such a definition of the diffraction limit is based on the spatial depletion of LW pulses generated by "finite-time"' dynamic apertures. One can argue that a pulse enters its far-field region when most of the initially significant spatial spectral components are depleted. These spectral components obviously characterize the spatial distribution of the source elements on the aperture. Hence, the loss of information through the depletion of these components signals the beginning of the far-field region. For ultra-wideband pulses one is faced with the ambiguity of determining what are the initially "significant", spatial spectral components. Should one use a 3-dB point as the highest "significant" frequency, or would a $\left(1 / e^{2}\right)$ or $\left(1 / e^{4}\right)$ point be a more suitable choice? This ambiguity reflects the fact that there is no sharp limit between the near- and far-field regions of ultra-wideband pulses. A definition of a diffraction limit can only be used to compare the relative performance of different LW pulses. The crucial issue here is to be consistent in our use of definitions of the radii and the bandwidths of the excitation wave fields. Since any of the above definitions can be used to specify the highest "significant" spatial spectral components, we shall use a generic cutoff frequency denoted by $\chi_{d}$. In this case, we may refer to the following expression for the diffraction length of LW pulses [cf. Eq. (12) in Ref. 7]:

$$
\begin{aligned}
Z_{d} & =\pi\left(\frac{4}{\eta(\omega / c)}\right)\left(\frac{\omega_{0}\left(\chi_{d}\right)}{\chi_{d} c}\right)^{2} \\
& =\mu\left(\chi_{d}\right) R_{\max } R_{\min }\left(\omega_{\max } / c\right) .
\end{aligned}
$$

Here, $\eta(\omega / c)$ is the effective width of the spectral $\omega$ window given in Eq. (17b) and $\omega_{0}\left(\chi_{d}\right)$ is the $\omega$ value of the center of $\hat{\delta}\left(\omega-\chi y_{0}\right)$ at $\chi=\chi_{d}$. The quantity $\mu\left(\chi_{d}\right)$ is a numerical factor of $O(1)$ that depends on the specific choice of $\chi_{d}$, in general. The diffraction length in Eq. (21) is expressed in terms of spectral quantities depicting the excitation wave field, ${ }^{7}$ or as a function of the dimensions of the utilized dynamic aperture and the bandwidth of its source elements. ${ }^{2,3}$ The two expressions may be deduced from each other if one recalls that $R_{\max }=R(2 T), R_{\min }=a_{1}$, and $\left(\omega_{\max } / c\right)=(4 \cosh \beta) / a_{1}$. Furthermore, one can choose the "significant" spatial components as those exhibiting amplitudes larger than $\left(1 / e^{4}\right)$ of the maximum of the spatial spectrum. As a consequence, explicit expressions for the diffraction length given in Eq. (21) can be derived. For the $\mathrm{X}$-wave pulse we define $\chi_{d}=4 / a_{1}, \eta(\omega / c)=4 / c T$, and $\omega_{0}\left(\chi_{d}\right)=c \chi_{d}$ $\cosh \beta$. In this case, Eq. (21) reduces to

$$
Z_{d}=\pi c T(\cosh \beta)^{2},
$$

which is independent of the choice of $\chi_{d}$. This means that the source of the $\mathrm{X}$-wave radiated field has the unique property of yielding a diffraction length that does not depend on the particular criterion chosen to define $\chi_{d}$. In contrast, the diffraction length of the time-limited FWM pulse is proportional to $\chi_{d}^{2}{ }^{7}$ Hence, $\mathrm{X}$ waves can serve to test the relative performance of other LW pulses of comparable source dimensions and temporal spectral bandwidths. For the parameter values used in Fig. 7, Eq. (22) gives $Z_{d}=64.25$, 128.5, 
and $192.75 \mathrm{~m}$. Since the maximum dynamic radius is given by $R_{\max }=2 c T \cosh \beta$, the diffraction limit given in Eq. (22) may be rewritten as

$$
Z_{d}=(\pi / 2) R_{\max } \cosh \beta=(\pi / 4) D \cosh \beta,
$$

where $D$ is the diameter of the source. Apart from a small difference in the numerical constant multiplying the $D \cosh \beta$ dependence of the diffraction limit, our result is the same as the one derived by $\mathrm{Lu}$ and Greenleaf. ${ }^{16-18}$ The difference in the numerical constant is due to the different criteria used to define the quantities involved in the two approaches.

The expression given in Eq. (21) illustrates the kind of performance enhancement that LW pulses exhibit when compared to quasi-monochromatic Gaussian wave fields. Consider, for example, the case of a quasi-monochromatic beam of waist $w_{0}=2 R_{\min }$ equal to that of the highly focused central portion of the $\mathrm{X}$ wave. For a static aperture having $D \gg w_{0}$, the associated diffraction length is defined as $Z_{w}$ $=\pi w_{0}^{2} / 4 \lambda_{\min }$. Using $\left(\omega_{\max } / c\right)=2 \pi / \lambda_{\min }$, the enhancement in the diffraction range of the $\mathrm{X}$ wave over an equivalent quasimonochromatic beam, of the same waist, is given by the ratio $\left(Z_{d} / Z_{w}\right) \propto\left(R_{\max } / R_{\min }\right)$. For the parameters used in this paper, the enhancement in the depth of propagation of the $\mathrm{X}$ wave over a comparable quasi-monochromatic signal is of order $O(10) \rightarrow O(40)$. This demonstrates that LW pulses become advantageous when narrow pulsed wave fields are required to have extended focused ranges. A dynamic aperture illuminated by a wave field having a time-dependent radius provides an efficient scheme to generate narrow LW pulses from much larger sources. Finally, one should note that the diffraction limit given in Eq. (22) is independent of the parameter $a_{1}$. This indicates that the diffraction length can be kept constant for pulses having different temporal bandwidths. As such, an X-wave pulse can travel to the same diffraction-free range even if we use an excitation wave field having a smaller bandwidth. This is true; however, the radius of the highly focused central portion of the radiated field is equal to $R_{\min }=a_{1}$ and $\omega_{\max } \propto 1 / a_{1}$. Hence, a smaller bandwidth corresponds to a larger focus radius $R_{\min }$ for which the enhancement ratio $\left(R_{\max } / R_{\min }\right)$ acquires smaller values. Consequently, the use of smaller bandwidths reduces the enhancement achieved over quasi-monochromatic signals.

\section{CONCLUDING REMARKS}

In this paper, we have demonstrated that an $\mathrm{X}$ wave exhibits all the features characterizing other LW pulses. It has been shown that it is characterized by the spatiotemporal spectral coupling that distinguishes LW pulses from other pulsed wave fields. ${ }^{6,7} \mathrm{~A}$ direct consequence of such a coupling is the unique depletion of the spectral components and, hence, the exhibited slow decay pattern of such LW pulses in the near-field range. The main difference between an X wave and other FWM-like wave fields is the functional dependence of the coupling between the temporal and spatial spectral components. Whereas the FWM displays an $\omega \propto \chi^{2}$ coupling, the $\mathrm{X}$ wave has an $\omega \propto \chi$ coupling. Once the similarity between an $X$ wave and other LW pulses is recognized, we can bring together the apparently disparate results of the two research areas into a single framework.

It has also been shown that an $\mathrm{X}$ wave may be generated from finite-time dynamic apertures analogous to those proposed as sources for launching FWM-like pulses. One should note that such sources acquire finite sizes by turning on/off their illumination wave fields at finite times. They exhibit most of the essential features of physical apertures and at the same time are much easier to handle mathematically. The illumination of the dynamic source of an $\mathrm{X}$ wave acquires the shape of an annulus whose size varies with time. This should be contrasted with the excitation of the FWM aperture that has the shape of a disc having a time-dependent radius. A finite-time dynamic aperture does not have a physical edge bounding its illumination wave field. This might shed some doubt on the validity of the results deduced using such a construction. To examine the origin of any possible discrepancies, we have studied the decay properties of an X wave generated from a finite radius source consisting of discrete concentric annular sections. The resulting decay patterns of the $\mathrm{X}$ wave have been shown to compare nicely with those derived using a finite-time dynamic source.

Similarly to other LW pulses generated from dynamic apertures, the spectral depletion of an $\mathrm{X}$ wave differs from conventional quasi-monochromatic pulsed wave fields. The decay in the spectral components contributing to the amplitude of the centroid of the propagating pulse results from the increasing oscillations introduced into the spectral $\omega$ windows with the distance $z$. One can, thus, utilize the knowledge gained from earlier investigations $\mathrm{s}^{2-7}$ to derive the diffraction limit characterizing an $\mathrm{X}$ wave. Our spectral depletion approach has established that an $\mathrm{X}$ wave has the attractive property that its diffraction length does not depend on the particular choice of $\chi_{d}$. As a consequence, the decay behavior of any other LW pulses can be compared to that of an $\mathrm{X}$ wave..$^{29}$ Both fields should have equal aperture sizes, with illumination wave fields exhibiting comparable bandwidths. Furthermore, the two radiated fields should attain equal focused waists. Finally, it has been shown that the enhancement in the focused depth of an X-wave dynamic source, in comparison to a static aperture used to generate quasi-monochromatic signals of a comparable waist is proportional to the ratio $\left(R_{\max } / R_{\min }\right)$.

${ }^{1}$ A. M. Shaarawi, R. W. Ziolkowski, and I. M. Besieris, "On the evanescent fields and the causality of the focus wave modes,' J. Math. Phys. 36, 5565-5587 (1995).

${ }^{2}$ A. M. Shaarawi, I. M. Besieris, R. W. Ziolkowski, and S. M. Sedky, “The generation of approximate focus wave mode pulses from wide-band dynamic Gaussian apertures,', J. Opt. Soc. Am. A 12, 1954-1964 (1995).

${ }^{3}$ A. M. Shaarawi, S. M. Sedky, R. W. Ziolkowski, and I. M. Besieris, "The spatial distribution of the illumination of dynamic apertures and its effect on the decay rate of the radiated localized pulses,' J. Phys. A 29, 51575179 (1996).

${ }^{4}$ R. W. Ziolkowski, I. M. Besieris, and A. M. Shaarawi, “Aperture realizations of the exact solutions to homogeneous-wave equations,', J. Opt. Soc. Am. A 10, 75-87 (1993).

${ }^{5}$ A. M. Shaarawi, S. M. Sedky, R. W. Ziolkowski, and F. M. Taiel, “'Effect of the switching pattern of the illumination of dynamic apertures on the ranges of the generated waves,', J. Opt. Soc. Am. A 13, 1712-1718 (1996)

${ }^{6}$ A. M. Shaarawi, S. M. Sedky, F. M. Taiel, R. W. Ziolkowski, and I. M. 
Besieris, "Spectral analysis of time-limited pulsed Gaussian wavefields," J. Opt. Soc. Am. A 13, 1817-1835 (1996).

${ }^{7}$ S. M. Sedky, A. M. Shaarawi, F. M. Taiel, and I. M. Besieris, "On the diffraction length of localized waves generated by dynamic apertures," J. Opt. Soc. Am. A 13, 1719-1727 (1996).

${ }^{8}$ R. W. Ziolkowski, "Localized transmission of electromagnetic energy," Phys. Rev. A 39, 2005-2033 (1989).

${ }^{9}$ R. W. Ziolkowski, "Localized wave physics and engineering," Phys. Rev. A 44, 3960-3987 (1991).

${ }^{10}$ R. W. Ziolkowski, I. M. Besieris, and A. M. Shaarawi, "Localized wave representations of acoustic and electromagnetic radiation," Proc. IEEE 79, 1371-1378 (1991)

${ }^{11}$ A. M. Vengsarkar, I. M. Besieris, A. M. Shaarawi, and R. W. Ziolkowski, "Closed-form localized wave solutions in optical fiber waveguides," J. Opt. Soc. Am. A 9, 937-949 (1992).

${ }^{12}$ A. M. Shaarawi, I. M. Besieris, and R. W. Ziolkowski, "Localized energy pulse trains launched from an open, semi-infinite, circular waveguide," J. Appl. Phys. 65, 805-813 (1989).

${ }^{13}$ J. N. Brittingham, "Focus wave modes in homogeneous Maxwell equations: transverse electric mode," J. Appl. Phys. 54, 1179-1189 (1983).

${ }^{14} \mathrm{R}$. W. Ziolkowski, "Exact solutions of the wave equation with complex source locations,"' J. Math. Phys. 26, 861-863 (1985).

${ }^{15}$ J. Durnin, "Exact solutions for nondiffracting beams. I. scalar theory," J. Opt. Soc. Am. A 4, 651-654 (1987).

${ }^{16}$ J. Y. Lu and J. F. Greenleaf, "Nondiffracting X waves-exact solutions to free space scalar wave equation and their finite aperture realization," IEEE Trans. Ultrason. Ferroelectr. Freq. Control 39, 19-31 (1992).

${ }^{17}$ J. Y. Lu, H. Zou, and J. F. Greenleaf, "AA new approach to obtain limited diffraction beams," IEEE Trans. Ultrason. Ferroelectr. Freq. Control 42, 850-853 (1995).
${ }^{18}$ J. Y. Lu and J. F. Greenleaf, "Experimental verification of nondiffracting X waves," IEEE Trans. Ultrason. Ferroelectr. Freq. Control 39, 441-446 (1992).

${ }^{19}$ J. Y. Lu and J. F. Greenleaf, "Sidelobe reduction for limited diffraction pulse-echo system," IEEE Trans. Ultrason. Ferroelectr. Freq. Control 40, 735-746 (1993).

${ }^{20}$ J. Y. Lu and J. F. Greenleaf, "Ultrasonic nondiffracting transducer for medical imaging,"' IEEE Trans. Ultrason. Ferroelectr. Freq. Control 37, 438-447 (1990)

${ }^{21}$ J. Y. Lu, M. Fatemi, and J. F. Greenleaf, "Pulsed-echo imaging with X wave," Acoust. Imaging 22, 191-196 (1996).

${ }^{22}$ J. Y. Lu and J. F. Greenleaf, "Diffraction-limited beams and their applications for ultrasonic imaging and tissue characterization," Proc. SPIE 1733, 92-119 (1992).

${ }^{23}$ J. Y. Lu, "Designing limited diffraction beams," IEEE Trans. Ultrason. Ferroelectr. Freq. Control 44, 181-193 (1997).

${ }^{24}$ F. Hsu, J. Margetan, and D. O Thomson, "Bessel beam ultrasonic transducer: Fabrication method and experimental results," Appl. Phys. Lett. 55, 2066-2068 (1989).

${ }^{25}$ D. Power, R. Donnelly, and R. MacIsaac, “'Spherical scattering of superpositions of localized waves,' Phys. Rev. E 48, 1410-1417 (1993).

${ }^{26}$ I. S. Gradshteyn and I. M. Ryzhik, Tables of Integrals, Series and Products (Academic, New York, 1965).

${ }^{27}$ P. M. Morse and H. Feshbach, Methods of Theoretical Physics (McGrawHill, New York, 1953), Sec. 11.3.

${ }^{28}$ B. Hafizi and P. Sprangle, "Diffraction effects in directed radiation beams,"' J. Opt. Soc. Am. A 8, 705-717 (1991).

${ }^{29}$ A. M. Shaarawi, "Comparison of two localized wave fields generated from dynamic apertures,’ J. Opt. Soc. Am. A 14, 1804-1816 (1997). 